\title{
Association of leprosy with HLA-DR2 in a Southern Brazilian population
}

J.E.L. Visentainer ${ }^{1}$ L.T. Tsuneto ${ }^{1}$ M.F. Serra ${ }^{2}$

P.R.F. Peixoto ${ }^{2}$ and M.L. Petzl-Erler ${ }^{3}$

\author{
'Departamento de Análises Clínicas, Universidade Estadual de Maringá, \\ 87020-900 Maringá, PR, Brasil \\ ${ }^{2}$ Centro de Especialidades do Sistema Único de Saúde, \\ 87013-280 Maringá, PR, Brasil \\ ${ }^{3}$ Departamento de Genética, Universidade Federal do Paraná, \\ 81531-990 Curitiba, PR, Brasil
}

\section{Correspondence \\ J.E.L. Visentainer \\ Departamento de Análises Clínicas Universidade Estadual de Maringá \\ Av. Colombo, 5790 \\ 87020-900 Maringá, PR \\ Brasil}

Research supported by Laboratório de Imunogenética and Mestrado em

Ciências Biológicas, Universidade Estadual de Maringá, and CNPq.

Received September 13, 1995 Accepted November 6, 1996

\begin{abstract}
The association between HLA specificities and leprosy was investigated in a Southern Brazilian population. One hundred and twentyone patients and 147 controls were typed for HLA-A, B, Cw, DR and DQ. Patients were subdivided into the following subgroups, according to clinical, histological and immunological criteria: lepromatous $(\mathrm{N}=55)$, tuberculoid $(\mathrm{N}=32)$, dimorphous $(\mathrm{N}=20)$, and indeterminate $(\mathrm{N}=14)$. The frequencies of HLA specificities were compared between the total group of patients and controls, and between the same controls and each subgroup of patients. After correction of the probabilities, deviations were not significant, except for the DR2 specificity, which presented a frequency of $44.2 \%$ in the total group of patients and $56.3 \%$ in the subgroup of individuals with the tuberculoid form of the disease, compared to $23.3 \%$ in the controls. Stratified analysis showed that the increased DR2 frequency in the total group of patients was due to the subgroups with the tuberculoid and dimorphous forms. The relative risk of tuberculoid leprosy for DR2-positive individuals was 4.2, and the etiologic fraction of DR2 was 0.429 . In conclusion, a positive association of the DR2 specificity with the tuberculoid form of leprosy, but not with the lepromatous, dimorphous, or indeterminate forms, was demonstrated in this Southern Brazilian population.
\end{abstract}

\section{Introduction}

Leprosy is a heterogeneous chronic infectious disease caused by the bacillus $M y$ cobacterium leprae (M. leprae) (1). The majority of people with leprosy live in tropical regions, but the disease also occurs in regions of temperate climate. It is believed that a long period of contact with $M$. leprae is necessary for infection to occur. The incubation period normally ranges from 2 to 5 years (2). The route of transmission of $M$. leprae is not known, but the upper airways, skin, and
Key words

- HLA

- Leprosy

- Mycobacterium leprae breast-feeding are likely routes.

Leprosy is still an important endemic disease in the great majority of countries of Asia, Africa, and Latin America. In 1991, the number of cases in the whole world was approximately 5.5 million, of which 270,000 were in Brazil (3). In adults, leprosy occurs at a proportion of 1.5 to 1.6 males to 1 female. In regard to age, in regions of high incidence, there is a peak in the second decade followed by a constant frequency in the period between 30 and 60 years (4).

Based on clinical, histological and immu- 
nological criteria, Ridley and Jopling (5) classified the leprosy spectrum into five groups: tuberculoid (TT), borderline-tuberculoid (BT), borderline-borderline (BB), borderline-lepromatous (BL) and lepromatous (LL). According to Seghal et al. (6), it would be convenient to add the indeterminate form (I) to this classification. The main signs of leprosy are hypoesthetic cutaneous lesions, dilatation of peripheral nerves and the presence of acid-resistant bacilli in skin lesions (2). The I form is an initial stage in which the clinical and histopathological outcome is uncertain. The lesions observed in the TT form are macular or infiltrated and may reappear or develop from indeterminate macules. In the LL form, the skin lesions are multiple and numerous bacilli can be demonstrated in skin lesion biopsies (7). LL may develop from the indeterminate or dimorphous (D) disease, or may be the first form recognized. Borderline (or dimorphous) leprosy is a mixture of several components of the TT and LL forms.

The clinical manifestations of leprosy and their severity seem to depend on the interaction between $M$. leprae and the immune system of the infected host. There is a good correlation between the cell-mediated immunity (CMI) against $M$. leprae antigens observed in vitro and in vivo and the clinical course of the disease. In the localized and less serious TT form, an efficient CMI to $M$. leprae develops with low levels of antibodies. The LL form, on the other hand, is associated with a flaw in CMI and patients have high antibody levels and a disseminated bacterial increase. High levels of antibodies in the LL form may be a consequence of a high bacillar charge (8), but these antibodies do not provide any protection against M. leprae (9). The I and D forms of the disease show various degrees of CMI and different levels of antibodies.

The phenotype of susceptibility to infection by M. leprae is complex and is influenced by a variety of both host and parasitic factors, and by the environmental conditions. The possible role of genetic factors of the host has been considered for many years. In 1929, Hopkins and Denny (10) postulated genetic variability as a basis for the epidemiologic observations of familial clustering and racial differences in the expression and incidence of the disease. In mice, the resistance or susceptibility to infection by many kinds of intracellular pathogens is controlled by the $B c g$ locus of chromosome 1 (11), while the haplotypes of the $\mathrm{H}-2$ system (the murine MHC) result in variability of the immune response to Mycobacterium lepraemurium (12). Based on the murine model, it has been suggested that a similar mechanism of genetic susceptibility should be operating in man $(7,13)$.

Many investigators have tried to find an association between leprosy and its subtypes with genetic markers, with the purpose of identifying susceptibility genes. More attention has been given to the HLA system, because alleles of its highly polymorphic loci encode class I and II HLA molecules, crucial to cellular interactions in CMI.

Earlier studies have reported increased frequencies of HLA-DR2 and DR3 in TT patients (14-21) and of HLA-DQ1 and DR2 in LL patients $(18,22-24)$, with interpopulation heterogeneity. Association studies between leprosy and class II HLA genes have not been performed in Brazilian populations.

The aim of the present study was to investigate the possible association between HLA and leprosy and/or its subtypes in a Southern Brazilian population. Therefore, the frequencies of the HLA phenotypes of 121 patients were compared to those of 147 normal individuals from the same ethnic group.

\section{Material and Methods}

\section{Patients and controls}

Patients with leprosy from the Regional Center for Specialties of the Unified Health 
System of the State of Paraná were selected and classified into four distinct subgroups according to Ridley and Jopling (5), including the I form (6). Fifty-five patients presented the LL form, 32 the TT form, 20 the D form and 14 the I form. Patients with the dimorphous form were classified into one group (D) due to the difficulty to differentiate the BT, BB, and BL forms and because of the reduced number of patients. After the exclusion of 3 consanguineous individuals, 118 individuals formed the total group of patients.

Clinical and laboratory diagnoses were established by the observation of the clinical signs of the disease by an experienced dermatologist, and by laboratory tests including bacilloscopy of the earlobes and elbows, skin biopsy and Mitsuda's reaction. The patients' response to the skin test with lepromin (killed M. leprae), known as Mitsuda's reaction, helps mainly in the classification of leprosy. After inoculation, the individual develops a granulomatous delayed type hypersensibility reaction. The TT patients were highly positive (reaction diameter $>5$ $\mathrm{mm}$ ) and the LL and D patients were negative $(0-2 \mathrm{~mm})$. Moreover, 12 individuals with the I form were negative while 2 patients were positive.

The control group consisted of 147 normal unrelated individuals, selected according to ethnic features, age, sex, occupation and geographical origin of the patients. Only individuals with Caucasoid morphological features were included in this study. The occupation of the great majority of the controls and patients was related to farming.

The M/F ratio and average age of the patients were 1.2 and $46.0 \pm 15.2$ years, respectively, and 1.3 and $44.5 \pm 14.0$ years for the controls. In the subgroups of patients, these parameters were 2.7 and $49.8 \pm 13$ for LL, 0.7 and $42.2 \pm 17.7$ for TT, 0.8 and 49.9 \pm 14.7 for subgroup $\mathrm{D}$, and 0.8 and $34.5 \pm$ 8.0 for the patients with the I form.

\section{HLA typing}

One hundred and twenty patients and 145 controls were typed for HLA-A, B and $\mathrm{Cw}$, while 116 patients and 146 controls were typed for HLA-DR. Among the latter, 116 patients and 145 controls were also typed for HLA-DQ. Some control individuals were not typed for all the specificities: the size of the samples is shown in Tables 1 to 5 .

Typing was performed according to the microlymphocytotoxicity method (25). The proportion of dead cells was evaluated by cytofluorochromasia (26). Three hundred anti-HLA antisera were used to type the following specificities: A1, A2, A3, A9, A23, A24, A10, A25, A26, A11, A28, A29, A30, A31, A33, A34, A36, B5, B51, B52, B7, B8, B12, B44, B45, B13, B14, B64, B65, B15, B62, B63, B16, B38, B39, B17, B18, B21, B49, B50, B22, B27, B35, B37, B40, B60, B61, B41, B42, B70, Cw1, Cw2, Cw3, Cw4, Cw5, Cw6, Cw7, Cw8, DR1, DR2, DR3, DR4, DR5, DR11, DR12, DR6, DR7, DR8, DR9, DR10, DR52, DR53, DQ1, DQ2, DQ3, DQ7 and DQ8/9.

\section{Statistical analysis}

Classical methods to study the association between HLA and disease (27) were used to detect differences in the frequencies of HLA specificities between patients and controls. The chi-square test was used, with Yates' correction (28) when needed. The P values were multiplied by the number of independent comparisons made (i.e. 66), resulting in the corrected P value or PC (29). The relative risks (RR) were calculated according to Woolf's method (30).

The etiologic fraction (EF) of the population was estimated according to Miettinen (31) and Green (32). EF indicates the proportion of disease cases attributable to a marker in the population (in this case, an HLA antigen), which presents a positive association $(R R>1)$ with the disease, and/or 
with other risk factors associated with this marker.

\section{Results}

The frequencies of HLA-DR and DQ specificities of the control group and of the total group of patients are listed in Table 1 . The frequencies of the same group of controls and the LL, TT, D, and I subgroups of patients are compared in Tables 2, 3, 4, and 5 , respectively. The frequencies of HLA-A, $\mathrm{B}$ and $\mathrm{CW}$ specificities were not included in the tables and they will be mentioned below only for those presenting the greatest deviations.

The following class I specificities had increased frequencies in the patients in comparison with the control group: HLA-A33 (patients $=4.3 \%$, control $=0.0 \% ; \mathrm{P}=0.0395)$, B5 (patients $=27.4 \%$, control $=17.2 \% ; \mathrm{P}=$ 0.0487 ), B51 (patients $=26.5 \%$, control $=$ $12.1 \% ; \mathrm{P}=0.0102$ ), $\mathrm{B} 7$ (patients $=18.8 \%$, control $=9.0 \% ; \mathrm{P}=0.02), \mathrm{Cw} 4$ (patients $=$ $36.8 \%$, control $=25.5 \% ; \mathrm{P}=0.0496)$ and $\mathrm{Cw} 7$ (patients $=46.2 \%$, control $=31.7 \% ; \mathrm{P}=$

Table 1 - HLA-DR and DQ antigen frequencies in the total sample of patients with leprosy and in the control group.

$\mathrm{ns}=$ Nonsignificant (indicated only if $\mathrm{P}<0.05$ before correction); $\mathrm{P}=$ probability; $\mathrm{PC}=$ corrected probability (Chi-square test).

\begin{tabular}{|c|c|c|c|c|c|c|c|}
\hline \multirow[t]{2}{*}{ Specificity } & Patients & Control & PC & Specificity & Patients & Control & PC \\
\hline & $(\%)$ & $(\%)$ & & & $(\%)$ & $(\%)$ & \\
\hline & $N=113$ & $N=146$ & & & $N=113$ & $N=92$ & \\
\hline DR1 & 23.9 & 16.4 & & DR10 & 4.4 & 2.2 & \\
\hline DR2 & 44.2 & 23.3 & 0.0231 & & $N=113$ & $N=145$ & \\
\hline DR3 & 15.0 & 18.5 & & DQ1 & 77.0 & 58.6 & $\mathrm{~ns}$ \\
\hline DR4 & 10.6 & 24.0 & ns & DQ2 & 23.0 & 36.6 & ns \\
\hline DR5 & 48.7 & 45.9 & & DQ3 & 76.1 & 61.4 & ns \\
\hline DR11 & 40.7 & 31.5 & & & $N=113$ & $N=91$ & \\
\hline DR12 & 7.1 & 13.0 & & DQ7 & 61.9 & 44.0 & $\mathrm{~ns}$ \\
\hline DR6 & 8.0 & 13.0 & & DQ8/9 & 8.0 & 14.3 & \\
\hline DR7 & 21.2 & 26.0 & & & & & \\
\hline DR8 & 3.5 & 2.7 & & & & & \\
\hline DR9 & 5.3 & 4.8 & & & & & \\
\hline DR52 & 69.9 & 68.5 & & & & & \\
\hline DR53 & 37.2 & 47.3 & & & & & \\
\hline
\end{tabular}

0.0168). A decrease was observed in the frequencies of HLA-A9 (patients $=17.1 \%$, control $=31.0 \% ; \mathrm{P}=0.0094), \mathrm{A} 23$ (patients $=3.4 \%$, control $=11.0 \% ; \mathrm{P}=0.021), \mathrm{B} 14$ (patients $=5.1 \%$, control $=13.8 \% ; \mathrm{P}=$ 0.0197 ) and B65 (patients $=3.4 \%$, control $=$ $14.3 \% ; \mathrm{P}=0.00454)$. However, the corrected $\mathrm{P}$ values $(\mathrm{PC})$ resulted in probabilities higher than 0.05 for all class I and class II antigens except for HLA-DR2 $(\mathrm{PC}=0.0231)$ (Table 1). The RR was 2.6 for DR2-positive individuals and the EF was 0.272.

Regarding the LL patients, an increase in the frequencies of HLA-B5 (patients $=31.5 \%$, control $=17.2 \% ; \mathrm{P}=0.0286)$ and B51 (patients $=25.9 \%$, control $=12.1 \% ; \mathrm{P}=0.033$ ) was observed. However, the deviations became nonsignificant after correcting the probabilities.

In the TT group, the following class I specificities presented increased frequencies: HLA-B5 (patients $=40.6 \%$, control $=17.2 \%$; $\mathrm{P}=0.00355), \mathrm{B} 51$ (patients $=34.4 \%$, control $=12.1 \% ; \mathrm{P}=0.00466), \mathrm{B} 7$ (patients $=28.1 \%$, control $=9.0 \% ; \mathrm{P}=0.00742)$ and $\mathrm{Cw} 7(\mathrm{pa}-$ tients $=56.3 \%$, control $=31.7 \% ; \mathrm{P}=$ 0.00896), while the frequency for HLA-Cw3 (patients $=6.35 \%$, control $=22.86 \% ; \mathrm{P}=$ 0.0338 ) was decreased. Again, these deviations were nonsignificant after correcting the probabilities, except for DR2 (PC = 0.0132) (Table 3). The calculated RR and the EF for DR2 were 4.2 and 0.429 , respectively.

The subgroup of patients with the $\mathrm{D}$ form of leprosy showed an increase in the frequencies of the following class I specificities: HLA-A1 (patients $=35.0 \%$; control $=$ $13.1 \% ; \mathrm{P}=0.0284), \mathrm{A} 33$ (patients $=10.0 \%$, control $=0.0 \% ; \mathrm{P}=0.00612)$ and $\mathrm{Cw} 7$ (patients $=55.0 \%$, control $=31.7 \% ; \mathrm{P}=0.0402$ ), while those with the I form presented an increase in the frequencies of HLA-A28 (patients $=28.6 \%$, control $=7.6 \% ; \mathrm{P}=0.0369)$ and $\mathrm{Cw} 7$ (patients $=64.3 \%$, control $=31.7 \%$; $\mathrm{P}=0.0314)$. After correcting the probabilities, none of the deviations were significant. 


\section{Discussion}

To determine a possible association between HLA specificities and leprosy and/or its clinical forms in a Southern Brazilian population, frequencies of HLA specificities were compared between a group of patients and a group of controls, and between the same controls and each subgroup of patients. Significant associations with the HLA specificity DR2 were shown for leprosy per $s e$ and for the tuberculoid form. However, it has been suggested that leprosy per se is not controlled by MHC-linked genes in either humans or mice $(7,12)$. Familial cosegregation and linkage analysis have supported the suggestion that not the susceptibility to leprosy, but the host response and the clinical type of leprosy are influenced by HLA-related factors (33). These and other studies indicate that the susceptibility to leprosy per $s e$ is controlled by other autosomal, nonMHC-linked genes, while HLA diversity is related to the subtype of leprosy which develops in patients after establishment of the infection (7). An explanation for the association observed in the present study is that it might ensue from the patient sample constitution, which comprises individuals with four different forms of leprosy. In the subgroup with the D form, 10 patients $(52.6 \%)$ were DR2-positive, while in the subgroup with the TT form, 18 patients $(56.3 \%)$ had this specificity. This contributes to the increase in the frequency of DR2 in the total sample of patients, since $44 \%$ of the total group of patients belong to these two groups. Frequencies of this specificity were analyzed after excluding individuals with the TT and/ or D forms. After excluding the TT and D forms, the frequencies of DR2 in the samples of patients (35.5\%) and controls (23.3\%) did not differ significantly $(\mathrm{P}=0.0697)$. This means that primarily the TT and D subgroups are contributing to the increased frequency of DR2 in the total sample of patients.
The analysis of the LL form of leprosy showed no significant association. Previous studies also reported a lack of significant associations between class I HLA antigens and this form of the disease (23). Regarding

\begin{tabular}{|c|c|c|c|c|c|c|c|}
\hline \multirow[t]{2}{*}{ Specificity } & \multirow{2}{*}{$\frac{\text { Patients }}{(\%)}$} & \multirow{2}{*}{$\frac{\text { Control }}{(\%)}$} & \multirow[t]{2}{*}{ PC } & \multirow[t]{2}{*}{ Specificity } & \multirow{2}{*}{$\frac{\text { Patients }}{(\%)}$} & \multirow{2}{*}{$\frac{\text { Control }}{(\%)}$} & \multirow[t]{2}{*}{ PC } \\
\hline & & & & & & & \\
\hline & $N=51$ & $N=146$ & & & $N=51$ & $N=92$ & \\
\hline DR1 & 21.6 & 16.4 & & DR10 & 2.0 & 2.2 & \\
\hline DR2 & 33.3 & 23.3 & & & $N=51$ & $N=145$ & \\
\hline DR3 & 13.7 & 18.5 & & DQ1 & 68.6 & 58.6 & \\
\hline DR4 & 9.8 & 24.0 & ns & DQ2 & 23.5 & 36.6 & \\
\hline DR5 & 56.9 & 45.9 & & DQ3 & 80.4 & 61.4 & ns \\
\hline DR11 & 45.1 & 31.5 & & & $N=51$ & 91 & \\
\hline DR12 & 9.8 & 13.0 & & DQ7 & 68.6 & 44.0 & ns \\
\hline DR6 & 13.7 & 13.0 & & DO8/9 & 5.9 & 14.3 & \\
\hline DR7 & 17.7 & 26.0 & & & & & \\
\hline DR8 & 5.9 & 2.7 & & & & & \\
\hline DR9 & 5.9 & 4.8 & & & & & \\
\hline DR52 & 80.4 & 68.5 & & & & & \\
\hline DR53 & 33.3 & 47.3 & & & & & \\
\hline
\end{tabular}

Table 3 - HLA-DR and DQ antigen frequencies in patients with the TT form of leprosy and in the control group.

$\mathrm{ns}=$ Nonsignificant (indicated only if $\mathrm{P}<0.05$ before correction); $\mathrm{P}=$ probability; $\mathrm{PC}=$ corrected probability (Chi-square test).

\begin{tabular}{|c|c|c|c|c|c|c|c|}
\hline \multirow[t]{2}{*}{ Specificity } & \multirow{2}{*}{$\frac{\text { Patients }}{(\%)}$} & \multirow{2}{*}{$\frac{\text { Control }}{(\%)}$} & \multirow[t]{2}{*}{ PC } & \multirow[t]{2}{*}{ Specificity } & \multirow{2}{*}{$\frac{\text { Patients }}{(\%)}$} & \multirow{2}{*}{$\frac{\text { Control }}{(\%)}$} & \multirow[t]{2}{*}{$\mathrm{PC}$} \\
\hline & & & & & & & \\
\hline & $N=32$ & $N=146$ & & & $N=32$ & $N=92$ & \\
\hline DR1 & 15.6 & 16.4 & & DR10 & 3.1 & 2.2 & \\
\hline DR2 & 56.3 & 23.3 & 0.0132 & & $N=51$ & $N=145$ & \\
\hline DR3 & 18.8 & 18.5 & & DQ1 & 78.1 & 58.6 & $\mathrm{~ns}$ \\
\hline DR4 & 6.3 & 24.0 & ns & DO2 & 28.1 & 36.6 & \\
\hline DR5 & 59.4 & 45.9 & & DQ3 & 81.3 & 61.4 & $\mathrm{~ns}$ \\
\hline DR11 & 53.1 & 31.5 & ns & & $N=32$ & $N=91$ & \\
\hline DR12 & 6.3 & 13.0 & & DQ7 & 65.6 & 44.0 & ns \\
\hline DR6 & 3.1 & 13.0 & & DQ8/9 & 6.3 & 14.3 & \\
\hline DR7 & 28.1 & 26.0 & & & & & \\
\hline DR8 & 3.1 & 2.7 & & & & & \\
\hline DR9 & 3.1 & 4.8 & & & & & \\
\hline DR52 & 71.9 & 68.5 & & & & & \\
\hline DR53 & 37.5 & 47.3 & & & & & \\
\hline
\end{tabular}


class II specificities, Izumi et al. (18) found a positive association of DQ1 with the LL form in Japan. An increase in DQ1 was also found in Venezuela (22) and in three different Asian countries (reviewed in Ref. 23). Recently, an increase in the frequencies of B60, DR2, DR8 and DQ1 in LL patients

Table 4 - HLA-DR and DQ antigen frequencies in patients with the D form of leprosy and in the control group.

$\mathrm{ns}=$ Nonsignificant (indicated only if $\mathrm{P}<0.05$ before correction); $\mathrm{P}=$ probability; $\mathrm{PC}=$ corrected probability (Chi-square test).

\begin{tabular}{|c|c|c|c|c|c|c|c|}
\hline \multirow[t]{2}{*}{ Specificity } & \multirow{2}{*}{$\frac{\text { Patients }}{(\%)}$} & \multirow{2}{*}{$\frac{\text { Control }}{(\%)}$} & \multirow[t]{2}{*}{ PC } & \multirow[t]{2}{*}{ Specificity } & \multirow{2}{*}{$\frac{\text { Patients }}{(\%)}$} & \multirow{2}{*}{$\frac{\text { Control }}{(\%)}$} & \multirow[t]{2}{*}{ PC } \\
\hline & & & & & & & \\
\hline & $N=19$ & $N=146$ & & & $N=19$ & $N=92$ & \\
\hline DR1 & 21.1 & 16.4 & & DR10 & 5.3 & 2.2 & \\
\hline DR2 & 52.6 & 23.3 & ns & & $N=19$ & $N=145$ & \\
\hline DR3 & 21.1 & 18.5 & & DQ1 & 73.7 & 58.6 & \\
\hline DR4 & 10.5 & 24.0 & & DO2 & 21.1 & 36.6 & \\
\hline DR5 & 36.8 & 45.9 & & DQ3 & 73.7 & 61.4 & \\
\hline DR11 & 31.6 & 31.5 & & & $N=19$ & $N=91$ & \\
\hline DR12 & 5.3 & 13.0 & & DQ7 & 57.9 & 44.0 & \\
\hline DR6 & 5.3 & 13.0 & & DQ8/9 & 10.5 & 14.3 & \\
\hline DR7 & 21.1 & 26.0 & & & & & \\
\hline DR8 & 0.0 & 2.7 & & & & & \\
\hline DR9 & 5.3 & 4.8 & & & & & \\
\hline DR52 & 63.2 & 68.5 & & & & & \\
\hline DR53 & 36.8 & 47.3 & & & & & \\
\hline
\end{tabular}

Table 5 - HLA-DR and DQ antigen frequencies in patients with the I form of leprosy and in the control group.

$\mathrm{ns}=$ Nonsignificant (indicated only if $\mathrm{P}<0.05$ before correction); $\mathrm{P}=$ probability; $\mathrm{PC}=$ corrected probability (Chi-square test).

\begin{tabular}{|c|c|c|c|c|c|c|c|}
\hline \multirow[t]{2}{*}{ Specificity } & Patients & Control & PC & Specificity & Patients & Control & PC \\
\hline & $(\%)$ & $(\%)$ & & & $(\%)$ & $(\%)$ & \\
\hline & $N=14$ & $N=146$ & & & $N=14$ & $N=92$ & \\
\hline DR1 & 50.0 & 16.4 & ns & DR10 & 14.3 & 2.2 & \\
\hline DR2 & 35.7 & 23.3 & & & $N=14$ & $N=145$ & \\
\hline DR3 & 14.3 & 18.5 & & DQ1 & 92.9 & 58.6 & ns \\
\hline DR4 & 28.6 & 24.0 & & DQ2 & 21.4 & 36.6 & \\
\hline DR5 & 21.4 & 45.9 & & DQ3 & 57.1 & 61.4 & \\
\hline DR11 & 14.3 & 31.5 & & & $N=14$ & $N=91$ & \\
\hline DR12 & 7.1 & 13.0 & & DQ7 & 42.9 & 44.0 & \\
\hline DR6 & 0.0 & 13.0 & & DQ8/9 & 14.3 & 14.3 & \\
\hline DR7 & 14.3 & 26.0 & & & & & \\
\hline DR8 & 0.0 & 2.7 & & & & & \\
\hline DR9 & 7.1 & 4.8 & & & & & \\
\hline DR52 & 42.9 & 68.5 & & & & & \\
\hline DR53 & 50.0 & 47.3 & & & & & \\
\hline
\end{tabular}

from the North of India was described (24). According to Mutis et al. (34), a specific peptide-HLA class II combination may exclusively activate Ts cells in lepromatous leprosy patients. The HLA-DR molecule encoded by allele HLA-DRB $1 * 1503$ (a recently discovered variant of HLA-DR2) binds and presents a peptide of mycobacterial hsp65 to Ts cells only. Different antigens that stimulate nonsuppressive $\mathrm{T}$ cells were presented by various HLA-DR2 alleles. Nishimura et al. (35) suggested that DQ molecules may control the immune response through the induction of CD8+ T suppressor cells.

In TT patients, differences from controls in the distribution of class I HLA specificities have been shown in many populations (reviewed in Ref. 23). In the great majority of these studies, as in the present one, these associations became nonsignificant when the probabilities were corrected. The strongest and more consistent associations were related to class II HLA specificities, mainly DR. In our population, the association between the HLA-DR2 specificity and the TT form of leprosy was confirmed. An association between HLA-DR2 and the TT clinical form was observed in a family study in India (15) and was confirmed afterwards in the same population (14). The same association was reported in other populations (18-21). Recently, an increase in the frequency of DRB1*1502 was reported in patients with TT leprosy, suggesting the involvement of this subtype of HLA-DR2 in pathogenesis (36). On the other hand, HLA-DR3 was associated with the TT form of leprosy in Surinam (16) and this was confirmed in Venezuelan families (22). The observation that susceptibility to TT leprosy is associated with DR2 and/or DR3 might be explained by genetic heterogeneity of these molecules in different populations, since particular alleles of HLA specificity (e.g. of DR2 and DR3) presented in one population might be absent in another one. In such a case, even postulating a direct involvement of HLA 
molecules in pathogenesis, different alleles might be responsible for the susceptibility of different populations. Alternatively, this discrepancy could be explained by the action of susceptibility allele(s) of other MHC genes in linkage disequilibrium with a DR2 allele in some populations and with a DR3 allele in other populations.

Anderson et al. (37) identified an $M$. leprae epitope in a $65-\mathrm{kDa}$ protein of the bacillus, which is restricted to HLA-DR2 and cross-reacts with a DR2 peptide. This particular epitope may be recognized only by $\mathrm{T}$ cells from DR2-positive individuals. $\mathrm{T}$ cell clones reacting against this peptide were obtained from individuals with the TT form of leprosy. The immune response of these patients is responsible for the immunopathological lesions which result in neural destruction. Thus, cross-reactivity between $M$. leprae and DR2 may play a role in the pathogenesis and immunopathology observed in tuberculoid leprosy. If the DR2 association with TT leprosy is due to a particular DR2 subtype has still to be proven. Rani et al. (38) studied allele frequency in patients with leprosy from North India using PCR-SSO typing. Both of the DR2 alleles commonly occurring in the Indian population, DRB $1 * 1501$ and DRB $1 * 1502$, were increased in the patients with leprosy, presenting a joint frequency of $81.5 \%$ in LL and BL patients, $60.7 \%$ in the TT group, and $21.3 \%$ in controls. However, this study is not informative regarding other DR2 alleles, since their frequency in the general northern Indian population is close to zero.

An aspect which may explain the participation of the HLA specificities in the susceptibility to the clinical forms of leprosy is related to the existence of different $\mathrm{T}$ cell populations. According to Gillis (39), at the tuberculoid pole, the induction of both Th cell populations (Th1 and Th2) may lead to an efficient immune response and, conse- quently, to mycobacterium elimination. At the lepromatous pole, Th2 T cells may preferably be activated, producing high IL-5 levels which would inhibit IL- 2 and IFN- $\gamma$ production, preventing an adequate immune response against the pathogen. According to de Vries (40), certain $M$. leprae peptides presented by particular class II HLA molecules, for example DR2, may preferably be recognized by $\mathrm{T}$ cells responsible for DTH. This could explain the association between the TT form of leprosy and DR2.

The $\mathrm{D}$ and I forms are rarely investigated. Recently, an increase in the frequencies of the B52, DR9, and DQ7 specificities has been shown in D (more specifically BL) patients from the North of India (24). In the present study, the specificities which presented a tendency to a positive association were different. However, the D and I subsamples were too small to allow clear conclusions.

The results obtained in the present study showed a positive association between HLADR2 and the tuberculoid form of leprosy, while no significant association between HLA and the LL, D or I forms of leprosy was demonstrable. Analyses at the molecular level may identify the DR2 allele(s) associated with the tuberculoid form in the Brazilian population and may differentiate whether the primary association is with DR2 itself or with alleles of closely linked genes in linkage disequilibrium with HLA-DR2.

\section{Acknowledgments}

We wish to express our gratitude to all patients and blood donor volunteers without whose collaboration this work would not have been possible. Furthermore, we would like to thank the staff of the Centro Regional de Especialidades of Sistema Único de Saúde and of the Laboratório de Imunogenética of the Universidade Estadual de Maringá, Brazil, for technical assistance. 


\section{References}

1. Hansen A (1874). Bacillus leprae. Norsk Magazine for Laegevidenskaben, 9: 1-21.

2. Meyers WM (1992). Leprosy. Dermatologic Clinics, 10: 73-96.

3. Noordeen SK, Lopez Bravo L \& Sundaresan TK (1992). Estimated number of leprosy cases in the world. Bulletin of the World Health Organization, 70: 7-10.

4. Noussitou FM (1976). Leprosy in Children. World Health Organization, Geneva, 1128.

5. Ridley DS \& Jopling WH (1966). Classification of leprosy according to immunity: A five-group system. International Journal of Leprosy, 34: 255-273.

6. Seghal VN, Jain MK \& Srivastava G (1989). Evolution of the classification of leprosy. International Journal of Dermatology, 28: 161-167.

7. Schurr E, Morgan K, Gros P \& Skamene E (1991). Genetics of leprosy. American Journal of Tropical Medicine and Hygiene, 44: 4-11.

8. Saad MHF, Medeiros MA, Gallo MEN \& Fonseca LS (1991). Use of the anti-PGL-I antibody ELISA and the Mitsuda reaction in early diagnosis of leprosy. Brazilian Journal of Medical and Biological Research, 24: 801-805.

9. Rani R, Zaheer SA, Suresh NR, Walia R, Parida SK, Mukherjee A, Mukherjee R \& Talwar GP (1992). Association of HLA antigens with differential responsiveness to Mycobacterium $w$ vaccine in multibacillary leprosy patients. Journal of Clinical Immunology, 12: 50-55.

10. Hopkins R \& Denny OE (1929). Leprosy in the United States. Journal of the American Medical Association, 92: 191-198.

11. Skamene E, Gros P, Forget A, Patel PJ \& Nesbitt MN (1984). Regulation of resistance to leprosy by chromosome 1 locus in the mouse. Immunogenetics, 19: 117124.

12. Curtis J, Akuffo-Adu H \& Turk JL (1984). $\mathrm{H}-2$ linked genes which modify resistance of C57BL/10 mice to subcutaneous infection with Mycobacterium lepraemurium. Infection and Immunity, 46: 635-638.

13. Abel L, Demenais F, Baule MS, Blanc M, Muller A, Raffoux C, Millan J, Bois E, Babron MC \& Feingold N (1989). Genetic susceptibility to leprosy on a Caribbean island: Linkage analysis with fine markers. International Journal of Leprosy, 57: 465-471.
14. van Eden W, de Vries RRP, Mehra NK, Vaidya MC, D'Amaro J \& van Rood JJ (1980). HLA segregation of tuberculoid leprosy: Confirmation of the DR2 marker. Journal of Infectious Diseases, 141: 693701.

15. de Vries RRP, Mehra NK, Vaidya MC, Gupte MD, Khan PM \& van Rood JJ (1980). HLA-linked control of susceptibility to tuberculoid leprosy and association with HLA-DR types. Tissue Antigens, 16: 294-304.

16. van Eden $W$, de Vries RRP, D'Amaro J, Schreuder I, Leiker DL \& van Rood JJ (1982). HLA-DR associated genetic control of the type of leprosy in a population from Surinam. Human Immunology, 4: 343-350.

17. van Eden W, Gonzalez NM, de Vries RRP, Convit J \& van Rood JJ (1985). HLA-linked control of predisposition to lepromatous leprosy. Journal of Infectious Diseases, 151: 9-14.

18. Izumi S, Sugiyama K, Matsumoto $Y$ \& Ohkawa $S$ (1982). Analysis of the immunogenetic background of Japanese leprosy patients by the HLA systems. Vox Sanguinis, 42: 243-247.

19. Rea TH \& Terasaki PI (1980). HLA-DR antigens in tuberculoid and lepromatous leprosy. Leprosy Review, 51: 117-123.

20. Miyanaga K, Jugi TR, Maeda H, Nakajima S \& Kobayashi S (1981). Tuberculoid leprosy and HLA in Japanese. Tissue Antigens, 18: 331-334

21. Schauf $V$, Ryan $S$, Scollard D, Jonasson $O$, Brown A, Nelson K, Smith T \& Vithayasai $\checkmark$ (1985). Leprosy associated with HLADR2 and DQw1 in the population of Northern Thailand. Tissue Antigens, 26: 243247.

22. Ottenhoff THM, Gonzalez NM, de Vries RRP, Convit J \& van Rood JJ (1984). Association of HLA specificity LB-E12 (MB1, DC1, MT1) with lepromatous leprosy in a Venezuelan population. Tissue Antigens, 24: 25-29.

23. Serjeantson SW (1983). HLA and susceptibility to leprosy. Immunological Reviews, 70: 89-112.

24. Rani R, Zaheer SA \& Mukherjee R (1992). Do human leukocyte antigens have a role to play in differential manifestation of multibacillary leprosy? A study on multibacillary leprosy patients from North India. Tissue Antigens, 40: 124-127.
25. Terasaki PI \& McClelland JD (1964). Microdroplet assay of human serum cytotoxins. Nature, 204: 998-1000.

26. Bodmer WF \& Bodmer J (1977). Cytofluorochromasia. In: Ray JG, Hare DB \& Kayhoe DE (Editors), NIAD Manual of Tissue Typing Techniques. National Institutes of Health, Bethesda, 31-34.

27. Svejgaard A, Jersild C, Nielsen S \& Bodmer WF (1974). HLA-antigens and disease: statistical and genetical considerations. Tissue Antigens, 4: 95-105.

28. Levin J (1978). Estatística Aplicada a Ciências Humanas. 2nd edn. Harper \& Row do Brasil Ltda., São Paulo.

29. Grumet FC, Coukell A, Bodmer JG, Bodmer WF \& McDevitt HO (1971). Histocompatibility (HLA) antigens associated with systemic lupus erythematosus. New England Journal of Medicine, 285: 193196.

30. Woolf B (1955). On estimating the relation between blood group and disease. Annals of Human Genetics, 19: 251-253.

31. Miettinen OS (1974). Proportion of disease caused or prevented by a given exposure, trait or intervention. American Journal of Epidemiology, 99: 325-332.

32. Green A (1982). The epidemiologic approach to studies of association between HLA and disease. II. Estimation of absolute risks, etiologic and preventive fraction. Tissue Antigens, 19: 259-268.

33. de Vries RRP, Serjeantson SW \& Layrisse Z (1984). Leprosy. In: Albert ED, Baur MP \& Mayr WR (Editors), Histocompatibility Testing 1984. Springer Verlag, Berlin, 362367.

34. Mutis T, Cornelisse YE, Datema G, van Del Elsen P, Ottenhoff THM \& de Vries RRP (1994). Definition of a human suppressor T-cell epitope. Proceedings of the National Academy of Sciences, USA, 91: 9456-9460

35. Nishimura $Y$, Kamikawaji N, Fujisawa $K$, Yoshizumi H, Yasunami M, Kimura A \& Sasazuki T (1991). Genetic control of immune response and disease susceptibility by the HLA-DQ gene. Research of Immunology, 142: 459-466.

36. Mehra NK, Rajalingam R, Mitra DK, Taneja V \& Giphart MJ (1995). Variants of HLADR2/DR51 group haplotypes and susceptibility to tuberculoid leprosy and pulmonary tuberculosis in Asian Indians. International Journal of Leprosy, 63: 241-248. 
37. Anderson DC, van Schooten WCA, Barry ME, Janson AAM, Buchanan TM \& de Vries RRP (1988). A Mycobacterium leprae-specific human $T$ cell epitope cross-reactive with an HLA-DR2 peptide. Science, 242: 259-261.
38. Rani R, Fernandez-Viña MA, Zaheer KR \& Stastny P (1993). Study of HLA class II alleles by PCR oligotyping in leprosy patients from North India. Tissue Antigens, 42: 133-137.
39. Gillis W (1989). T-Helper cell subpopulations and the immune spectrum of leprosy. International Journal of Leprosy, 57: 73-81.

40. de Vries RRP (1991). An immunogenetic view of delayed type hypersensitivity. Tubercle, 72: 161-167. 